\title{
Cortisol in Peripheral Blood Predicts the Severity and Prognosis in Patients with Liver Failure at 90 Days
}

\author{
Jian Zhang' \\ Junfeng $\mathrm{Li}^{2}$ \\ Mei Ding' \\ Yu Chen' \\ Zhongping Duan' \\ 'Difficult \& Complicated Liver Diseases \\ and Artificial Liver Center, Beijing Youan \\ Hospital, Capital Medical University, \\ Beijing, 100069, People's Republic of \\ China; ${ }^{2}$ Department of Infectious \\ Diseases, The First Hospital of Lanzhou \\ University, Lanzhou, 730000, People's \\ Republic of China
}

\begin{abstract}
Objective: To investigate the cortisol level in peripheral blood in evaluating the severity and prognosis of patients with liver failure.

Methods: A total of 211 inpatients with liver failure were enrolled in Beijing You 'an Hospital affiliated with Capital Medical University, from September 2018 to January 2020. They were divided into three groups according to the severity of liver failure: early-stage liver failure, medium-stage liver failure and end-stage liver failure. In all patients, peripheral venous blood was gathered to detect the level of peripheral cortisol and related biochemical indexes. Logistic regression was used to screen the risk factors affecting the prognosis of patients at 90 days. A receiver operating curve (ROC) was used to reflect the value of cortisol on the prognosis of patients at 90 days. A model for end-stage liver disease (MELD) was accessed.

Results: Among these 211 patients with liver failure, aged from 19 to 70 years, the average level of cortisol was $351.26 \pm 129.78 \mathrm{nmol} / \mathrm{L}$ for 101 cases with early-stage liver failure, $281.05 \pm$ $81.31 \mathrm{nmol} / \mathrm{L}$ for 58 cases with medium-stage liver failure, and $189.21 \pm 89.27 \mathrm{nmol} / \mathrm{L}$ for 52 cases with end-stage liver failure $(P<0.05)$. The level of cortisol in the survival group and the non-survival group was $339.26 \pm 121.19 \mathrm{nmol} / \mathrm{L}$ and $192.71 \pm 67.23 \mathrm{nmol} / \mathrm{L}$, respectively $(P<$ $0.05)$. The results of logistics regression analysis showed that the odds ratio (OR) and $95 \%$ confidence intervals $(\mathrm{CI})$ of cortisol were 1.025 and $1.013-1.038 \mathrm{nmol} / \mathrm{L}(P<0.05)$. MELD results showed that patients with higher MELD scores had lower cortisol content.

Conclusion: The level of cortisol is correlated with the severity of liver failure and decreases with the aggravation of liver failure, suggesting that the level of cortisol could be used as one of the indicators to evaluate the prognosis in patients with liver failure at 90 days.
\end{abstract}

Keywords: liver, liver failure, prognosis, cortisol

\section{Introduction}

Liver failure, which remains a worldwide health problem, is a complex syndrome caused by a variety of factors, leading to severe disorder or decompensation of synthesis, detoxification, metabolism and biotransformation functions, with jaundice, coagulation dysfunction, hepatorenal syndrome, hepatic encephalopathy, and ascites as the main manifestations of a group of clinical syndromes. ${ }^{1-4}$ Many reasons cause liver failure. Hepatitis $\mathrm{B}$ virus (HBV) is the leading cause of liver failure in China, and the mortality rate ranges from $50 \%-80 \%{ }^{5-7}$ It has been reported that patients with liver failure have a very high mortality rate at 90 days of illness. ${ }^{8-10}$ At present, there is no effective treatment except liver transplantation. Liver transplantation could significantly reduce mortality in patients, but its clinical application is limited due to the lack of liver sources and its high cost. ${ }^{11-13}$ Therefore, an early and accurate assessment of patients with liver failure could
Correspondence: Zhongping Duan Difficult \& Complicated Liver Diseases and Artificial Liver Center, Beijing Youan Hospital, Capital Medical University,

No. 8, You An Men Outer Street, Fengta District, Beijing, 100069, People's

Republic of China

Tel +86-13466675/22

Email zhangdajian1978@ccmu.edu.cn 
provide the appropriate treatment for improving the prognosis of patients. Currently, the commonly used methods for evaluating the severity and prognosis of patients with liver failure are mostly based on the functions of multiple organs themselves. ${ }^{14-17}$ But this method has limitations in terms of accuracy and convenience of application. Hormones play an important role in body metabolism and organ function regulation. Previous studies have suggested that glucocorticoids may be helpful in the treatment of severe liver failure, which is often associated with adrenal failure. ${ }^{18}$ Cortisol is an endogenous glucocorticoid secreted by the adrenal cortex, and it is an important cholesterol derivative for maintaining life homeostasis. ${ }^{19}$ The concentration of corticosteroid-binding globulin decreased acutely, and its binding affinity with cortisol decreased. ${ }^{20}$ This suggests that baseline cortisol levels may serve as a prognostic indicator and may be used to determine the severity of liver failure. In this study, a prospective cohort observational study was conducted to detect the amount of cortisol in peripheral blood of patients with liver failure and to analyse the relationship between the amount of cortisol and the severity of liver failure. The purpose of this study was to provide a basis for predicting the 90-day prognosis of patients with liver failure with levels of cortisol.

\section{Materials and Methods Study Population}

A total of 225 patients with liver failure aged 19 to 70 years who were hospitalised in Beijing You 'an Hospital, Capital Medical University, from September 2018 to February 2020 were enrolled in this study. Inclusion criteria: in line with the definition of liver failure in the Guidelines for the Diagnosis and Treatment of Liver Failure (2018 Edition) formulated by the Chinese Medical Association ${ }^{1}$ (1) extreme fatigue and obvious gastrointestinal symptoms; (2) jaundice aggravated, total serum bilirubin (TBIL) was ten times higher than the upper limit of normal or daily increase of TBIL was $\geq 17.1$ $\mathrm{mmol} / \mathrm{L}$; (3) Patients with bleeding tendency, PTA $\leq 40 \%$ (or INR $\geq 1.5$ ); (4) decompensated ascites; (5) with or without hepatic encephalopathy. Exclusion criteria: (1) diagnosed by enhanced CT combined with serum markers; (2) left ventricular systolic insufficiency diagnosed by ultrasound Doppler examination; (3) renal insufficiency diagnosed according to serum creatinine $>133 \mathrm{umol} / \mathrm{L}$; (4) treated with glucocorticoid within three months. The present study was approved by Beijing You 'an Hospital (2019-989), affiliated with Capital
Medical University. An informed consent form was signed by all patients during their hospitalisation.

\section{Data Collection}

Clinical information of patients was collected, including gender, age, the basis of liver disease, and causes of liver failure (HBV reactivation, abdominal infection, alcohol consumption, upper gastrointestinal bleeding, respiratory infection, and drugs). Patients were followed up 90 days after admission. Outcomes (survival, death, and liver transplantation) were collected 90 days after diagnosis by an outpatient medical record system or telephone follow-up. Liver transplantation patients were considered lost to follow-up. Blood samples were collected from all patients at 6:00 am on the second day after admission and tested in the Clinical Laboratory Center of Beijing You 'an Hospital, Capital Medical University. Serum was obtained by centrifugation and then detected. Biochemical indicators: glutamate aminotransferase (ALT), aspartate aminotransferase (AST), TBIL, HBV-DNA quantification, serum creatinine (CR), albumin (ALB), serum sodium (NA). The detection instrument used was an automatic biochemical analyser (Olympus AU 5400 system, Japan), and the operation was carried out according to the laboratory operating procedures. Coagulation indicators: the international standardisation of prothrombin time (PT-INR) detection instrument was an automatic coagulation analyser (ACL Company Top 700).

\section{Determination of Serum Cortisol}

Serum cortisol was determined using a Roche E60 electrochemiluminescence analyser purchased from EnzymeLinked Bio Human Cortisol Kit (ELISA). Blood samples were left to clot for two hours at room temperature, protected from the light, and later centrifuged at $1000 \times \mathrm{g}$ for 20 minutes in the centrifuge. The serum samples, at least 250 $\mu \mathrm{L}$, aspired with an automatic pipette, were stored in sterile tubes positioned vertically at $-20^{\circ} \mathrm{C}$ to preserve the stability of cortisol. The cortisol concentration of the samples was quantified using an immunoassay kit and validated following the instructions provided by the manufacturer.

The specific operations were as follows: (1) all reagents were prepared before starting the assay procedure, and all standards and samples were added to the microtiter plate; (2) $50 \mu \mathrm{L}$ of standard or sample was added to the appropriate wells. Nothing was added to the blank well; (3) $100 \mu \mathrm{L}$ of enzyme conjugate was added to standard wells and sample wells except the blank well then covered with an adhesive strip and incubated for 60 
minutes at $37^{\circ} \mathrm{C}$; (4) the microtiter plate was washed four times; (5) $50 \mu \mathrm{L}$ of substrate $\mathrm{A}$ and $50 \mu \mathrm{L}$ of substrate $\mathrm{B}$ was added to each well. All were gently mixed and incubated for 15 minutes at $37^{\circ} \mathrm{C}$, protected from the light; (6) $50 \mu \mathrm{L}$ stop solution was added to each well; (7) Optical Density (OD) was read at $450 \mathrm{~nm}$ using a microtiter plate reader within 15 minutes.

\section{Diagnostic Criteria}

Early stage of liver failure: (1) extreme fatigue and obvious anorexia, vomiting, abdominal distension, and other serious gastrointestinal symptoms; (2) jaundice was progressive (serum TBIL $\geq 171 \mathrm{mmol} / \mathrm{L}$ or daily increase $\geq 17.1 \mathrm{mmol} / \mathrm{L}$ ); (3) bleeding tendency, $30 \%$ $<$ PTA $\leq 40 \%$ (or $1.5<\mathrm{INR} \leq 1.9$ ); (4) no hepatic encephalopathy or other complications occurred. Medium stage of liver failure: on the basis of the early manifestations of liver failure, and one of the following two: (1) II degrees of hepatic encephalopathy or ascites, or both, and infection; (2) significant bleeding tendency (bleeding points or ecchymosis), 20\% $<$ PTA $\leq 30 \%$ (or $1.9<$ INR $\leq 2.6$ ). End stage of liver failure: on the basis of liver failure medium-stage performance, the illness aggravating, severe bleeding tendency (injection site ecchymosis), the PTA $20 \%$ or less (or INR 2.6 or higher), and one of the following four: hepatorenal syndrome, upper gastrointestinal bleeding, severe infections, II above the degree of hepatic encephalopathy.

The model for end-stage liver disease (MELD) score was used to assess the liver dysfunction in patients with liver failure. According to the MELD score, the patients were divided into three groups: MELD $<30$ group, MELD $<40$ group, and MELD $>40$ group. The cortisol levels of the three groups were compared. The MELD score was calculated by the following formula:

$\operatorname{MELD}$ score $=3.8 \ln [$ TBIL $(\mathrm{mg} / \mathrm{d} 1)]+11.2+9.61 \mathrm{n} /$ $\mathrm{n}(\mathrm{INR}) / \mathrm{CR}(\mathrm{mg} / \mathrm{d} 1)+6.4$.

After stimulation with adrenocorticotropic hormone (ACTH), the low-level inappropriate response to adrenocortical hormone was called relative adrenal insufficiency (RAI), which was considered to be closely related to mortality. It is a concept used to describe the failure of adrenal secretion in critically ill patients under stress. RAI was diagnosed when the cortisol level was lower than 10 $\mathrm{ug} / \mathrm{dL}$ (lower than $248 \mathrm{nmol} / \mathrm{L}){ }^{21}$

\section{Statistical Analysis}

SPSS 25.0 statistical analysis software was used for statistical analysis. Measurement data conforming to normal distribution were expressed as mean \pm standard deviation $(\mathrm{X} \pm \mathrm{SD})$. Analysis of variance was used to compare the mean values of the three groups with different stages of liver failure and different MELD scores. Prognostic factors were analysed by univariate and multivariate logistic regression, and the area under the receiver operating characteristics (AUROC) was used to test the prognostic effect of cortisol levels. $P<$ 0.05 suggested a statistically significant difference.

\section{Results}

\section{Baseline Characteristics According to Liver Failure Group}

We excluded six cases of liver cancer, two cases of left ventricular systolic insufficiency, three cases of renal insufficiency and three cases treated with glucocorticoid within three months. Finally, 211 patients were included (Figure 1).

The results showed that $101(47.87 \%)$ cases had earlystage liver failure, $58(27.49 \%)$ cases had medium-stage liver failure, and 52 (24.64\%) cases had end-stage liver failure. A comparison of the general conditions of the three groups is shown in Table 1. Of the 211 patients, $178(84.4 \%)$ were male, with an average age of $45.7 \pm 10.4$ years. There were $33(15.6 \%)$ females with an average age of $56.3 \pm 11.7$ years. In terms of clinical manifestations, 87 (41.2\%) cases had chronic hepatitis, and 124 (58.8\%) cases had cirrhosis. From the course of the disease, four $(6.64 \%)$ cases had acute liver failure, $102(48.34 \%)$ cases had chronic and acute/ subacute liver failure, and $95(45.02 \%)$ cases had chronic liver failure. In terms of aetiology, $87(41.23 \%)$ cases had HBV, 31 (14.69\%) cases had hepatitis C, 48 (22.75\%) cases had alcoholic liver disease, 16 (7.58\%) cases had druginduced liver disease, and 29 (13.74\%) had autoimmune liver disease. Among the acute aggravating factors of liver

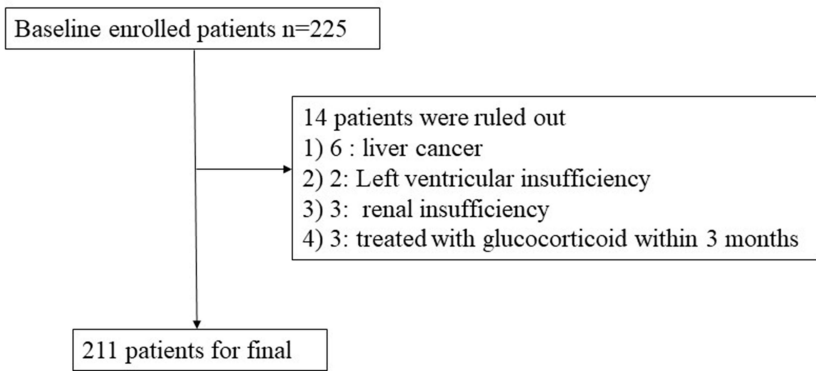

Figure I Diagrammatic sketch of the screening process. 
Table I Comparison of General Characteristics Between Different Stages of Liver Failure

\begin{tabular}{|c|c|c|c|c|}
\hline & Early-Stage ( $10 \mid$ Cases) & Medium-Stage (58 Cases) & End-Stage (52 Cases) & $P$-value \\
\hline Age, years & $48.13 \pm 13.36$ & $48.86 \pm 8.60$ & $44.06 \pm 8.71$ & 0.050 \\
\hline Male (\%) & 89 (88. $12 \%)$ & $44(75.86 \%)$ & 45 (86.54\%) & 0.011 \\
\hline Chronic hepatitis (\%) & $36(35.64 \%)$ & $25(43.10 \%)$ & $26(50.00 \%)$ & 0.000 \\
\hline HBV-reactivates & 10 & 12 & 16 & 0.000 \\
\hline Gastrointestinal bleeding & 3 & 2 & 7 & 0.000 \\
\hline Alcohol & 2 & 7 & 5 & 0.000 \\
\hline Spontaneous peritonitis & 6 & 11 & 12 & 0.000 \\
\hline Lung infection & $\mathrm{I}$ & 1 & 3 & 0.000 \\
\hline Drug & 0 & 3 & $\mathrm{I}$ & 0.000 \\
\hline TBil (mmol/L) & $292.57 \pm 169.32$ & $361.60 \pm 173.67$ & $462.88 \pm 187.08$ & 0.000 \\
\hline INR & $1.95 \pm 0.30$ & $2.92 \pm 0.62$ & $5.34 \pm 1.01$ & 0.000 \\
\hline ALB (g/L) & $31.96 \pm 3.87$ & $31.79 \pm 3.60$ & $32.08 \pm 4.34$ & 0.927 \\
\hline $\mathrm{Cr}(\mathrm{mmol} / \mathrm{dL})$ & $80.63 \pm 22.27$ & $75.54 \pm 24.53$ & $119.70 \pm 31.17$ & 0.006 \\
\hline $\mathrm{Na}(\mathrm{mmol} / \mathrm{L})$ & $135.26 \pm 4.17$ & $135.88 \pm 4.60$ & $134.88 \pm 6.85$ & 0.579 \\
\hline MELD score & $25.93 \pm 4.68$ & $30.33 \pm 3.55$ & $36.79 \pm 3.40$ & 0.000 \\
\hline Cortisol (nmol/L) & $351.26 \pm 129.78$ & $28 \mid .05 \pm 81.31$ & $|89.2| \pm 89.27$ & 0.000 \\
\hline RAl cases (\%) & $23(22.78 \%)$ & $23(39.66 \%)$ & 43 (82.69\%) & 0.000 \\
\hline
\end{tabular}

Note: $P<0.05$ was considered statistically significant.

Abbreviations: TBil, total bilirubin; INR, standardized ratio of prothrombin; ALB, plasma albumin; CR, serum creatinine; NA, serum sodium ions; MELD, model of endstage liver disease; RAI, relative adrenal cortical insufficiency.

failure, there were 38 cases of HBV reactivation (37.25\%), 29 cases of spontaneous peritonitis (28.43\%), 14 cases of drinking alcohol (13.73\%), 12 cases of gastrointestinal bleeding $(11.76 \%)$, five cases of pulmonary infection $(4.90 \%)$, and four cases of hepatotoxicity of drugs (3.92\%). There were 118 cases of hepatic encephalopathy (55.92\%), 28 cases of renal failure (13.27\%), 21 cases of pulmonary failure $(9.95 \%)$, and 12 cases of circulatory failure $(5.69 \%)$.

Patients with viral hepatitis received antiviral treatment according to clinical guidelines, and conventional treatment included liver protection therapy, plasma infusion, albumin, and nutritional support. There were 26 (12.32\%) patients who received artificial liver plasmapheresis. Patients with hepatorenal syndrome or renal failure receive albumin, terlipressin or renal replacement therapy, or both. Patients with hepatic encephalopathy were treated with lactulose, ornithine aspartate and arginine. Bacterial infections were treated with antibiotics. Shock patients were given antishock treatment, such as blood volume expansion and vasoactive drugs. Patients with respiratory failure received oxygen therapy and ventilator support when necessary. There were $18(8.53 \%)$ patients who received liver transplantation during hospitalisation.
As shown in Table 1, the cortisol level of patients with early-stage liver failure was $351.26 \pm 129.78 \mathrm{nmol} / \mathrm{L}$, and the RAI ratio was $22.78 \%$. The cortisol level of patients with medium-stage liver failure was $281.05 \pm 81.31 \mathrm{nmol} /$ $\mathrm{L}$, and the RAI ratio was $39.66 \%$. The cortisol level of patients with advanced liver failure was $189.21 \pm 89.27$ $\mathrm{nmol} / \mathrm{L}$, and the RAI ratio was $82.69 \%(P<0.05)$.

\section{Associate of Different MELD Scores with Cortisol Levels}

Figure 2 shows that in the group with a MELD score $<30$, the cortisol level was $354.34 \pm 122.64 \mathrm{nmol} / \mathrm{L}$. In the group with a $30<\mathrm{MELD}<40$, the cortisol level was 247.26 $\pm 91.13 \mathrm{nmol} / \mathrm{L}$. In the MELD $>40$ group, the cortisol level was $173.23 \pm 68.77 \mathrm{nmol} / \mathrm{L}(P<0.01)$.

\section{Comparison of Cortical Content Between Survival Group and Death Group}

The patients were divided into a survival group and a death group based on the prognosis of patients at 90 days. In the survival group, there were 143 cases (67.77\%), and 68 cases (32.23\%) had died. The details are shown in Table 2. 


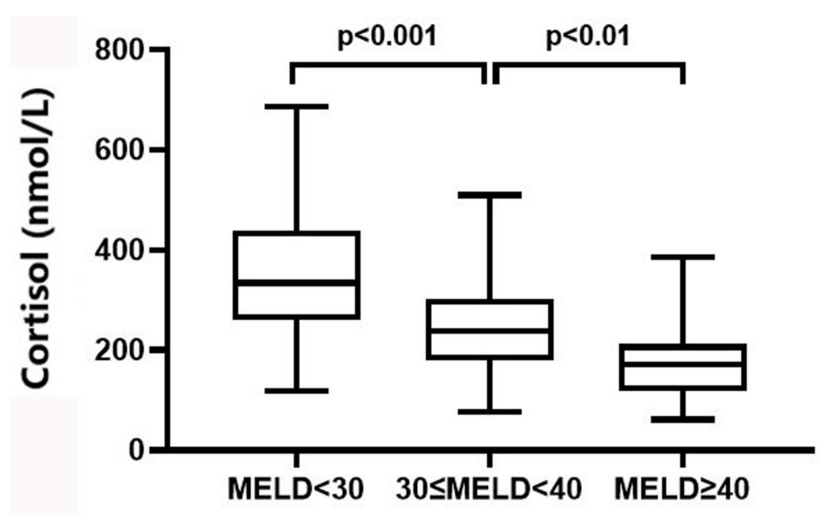

Figure 2 Comparison of cortisol levels in patients with different MELD score.

As shown in Table 2, the cortisol level of patients in the survival group was $339.26 \pm 121.19 \mathrm{nmol} / \mathrm{L}$, and the incidence of RAI was $23.78 \%$. The cortisol level of patients in the no-survival group was $192.71 \pm 67.23 \mathrm{nmol} / \mathrm{L}$, and the incidence of RAI was $80.88 \%(P<0.01)$. Cortisol levels in the no-survival group were significantly lower than those in the survival group.

\section{Univariate and Multivariate Logistic Regression Analysis of Risk Factors for Liver Failure}

We conducted the univariate and multivariate logistic regression analysis, and found that age $(\mathrm{OR}=0.890, P=0.002)$, MELD score $(\mathrm{OR}=0.718, P=0.017)$, TBIL $(\mathrm{OR}=0.993$, $P=0.005)$, and cortisol level $(\mathrm{OR}=1.025, P=0.000)$ was related to the prognosis of patients with liver failure admitted to hospital (Table 3). Age, MELD score, and TBIL were the independent protection factors. Cortisol level was an independent risk factor. The following formula was obtained by binary logistic regression analysis: $\mathrm{Y}=24.07+0.025$ (cortisol level) -0.331 (MELD score) -0.116 (age) -0.007 (TBIL).

\section{Level of Cortisol at 90-Day for Prognosis of Patients with Liver Failure}

The AUC of ROC curves about the prognosis of patients with liver failure is shown in Figure 3. The AUC of cortisol was $0.8525, P<0.001$. The cut-off values for the prediction of mortality were identified for cortisol levels $(252.3 \mathrm{nmol} / \mathrm{L}$, sensitivity $=0.762$, 1 -specificity $=0.176)$.

\section{Discussion}

The concept of "hepato-adrenal syndrome" was put forward by Marik et al in 2004 because of findings that showed $72 \%$ of patients with liver failure had adrenal insufficiency. $^{22}$ With the development of liver failure, the incidence of adrenocortical insufficiency gradually increases. It is proposed that cortisol levels are likely to correlate with the severity of liver failure and may indicate a poor prognosis. ${ }^{23}$ This provides a basis for the use of cortisol levels to assess disease severity and prognosis. Our results suggested that cortisol played a crucial role in the process and severity of liver failure, and the higher the level was, the higher the survival rate of patients was.

In this study, the incidence of RAI in patients with liver failure at admission was $42.18 \%$. It has been reported that the incidence of RAI in HBV-ACLF patients was $43 \%$, ${ }^{24}$

Table 2 Comparison of General Characteristics Between Survival Group and No-Survival Group

\begin{tabular}{|l|l|l|l|}
\hline & $\begin{array}{l}\text { Survival Group } \\
\text { (143 Cases) }\end{array}$ & $\begin{array}{l}\text { No-Survival Group } \\
\text { (68 Cases) }\end{array}$ & P-value \\
\hline Age, years & $41.10 \pm 13.36$ & $48.86 \pm 8.60$ & 0.041 \\
Male (\%) & $118(82.52 \%)$ & $60(88.24 \%)$ & 0.011 \\
Chronic hepatitis (\%) & $60(41.96 \%)$ & $27(39.71 \%)$ & 0.767 \\
TBil (mmol/l) & $278.48 \pm 148.13$ & $511.31 \pm 151.98$ & 0.000 \\
INR & $2.34 \pm 0.70$ & $4.51 \pm 1.62$ & 0.000 \\
ALB (g/L) & $32.06 \pm 3.90$ & $31.69 \pm 3.92$ & 0.613 \\
Cr (mmol/dL) & $66.61 \pm 22.56$ & $135.64 \pm 42.53$ & 0.000 \\
Na (mmol/L) & $135.67 \pm 4.17$ & $134.63 \pm 6.71$ & 0.239 \\
MELD score & $26.99 \pm 4.53$ & $35.76 \pm 4.05$ & 0.000 \\
Cortisol (nmol/L) & $339.26 \pm 121.19$ & $192.71 \pm 67.23$ & 0.000 \\
RAl cases (\%) & $34(23.78 \%)$ & $55(80.88 \%)$ & 0.000 \\
\hline
\end{tabular}

Note: $P<0.05$ was considered statistically significant.

Abbreviations: TBil, total bilirubin; INR, standardized ratio of prothrombin; ALB, plasma albumin; CR, serum creatinine; NA, serum sodium ions; MELD, model of endstage liver disease; RAI, relative adrenal cortical insufficiency. 
Table 3 Logistic Regression Analysis of Prognostic Independent Risk Factors for Patients 90 Days After Admission

\begin{tabular}{|l|c|l|l|l|}
\hline \multirow{2}{*}{} & \multicolumn{2}{|l|}{ Univariate Logistic Regression Analysis } & \multicolumn{2}{l|}{ Multivariate Logistic Regression Analysis } \\
\cline { 2 - 5 } & OR (95\% Cl) & $\underline{P}$-value & OR (95\% Cl) & $P$-value \\
\hline Age, years & $0.995(0.970-1.020)$ & 0.000 & $0.890(0.828-0.957)$ & 0.002 \\
Male (\%) & $0.629(0.268-1.479)$ & 0.288 & $0.368(0.050-2.70 I)$ & 0.325 \\
Chronic hepatitis (\%) & $1.098(0.609-1.977)$ & 0.756 & $1.258(0.589-9.801)$ & 0.369 \\
TBil (mmol/L) & $0.991(0.989-0.994)$ & 0.000 & $0.993(0.988-0.998)$ & 0.005 \\
INR & $0.301(0.202-0.446)$ & 0.000 & $0.263(0.044-1.555)$ & 0.141 \\
ALB (g/L) & $1.025(0.952-1.105)$ & 0.511 & $1.052(0.904-1.225)$ & 0.511 \\
Cr (mmol/dL) & $0.982(0.974-0.990)$ & 0.000 & $0.992(0.974-1.010)$ & 0.385 \\
Na (mmol/L) & $1.042(0.984-1.104)$ & 0.163 & $0.940(0.815-1.085)$ & 0.400 \\
Cortisol (nmol/L) & $1.019(1.014-10.25)$ & 0.000 & $1.025(1.013-1.038)$ & 0.000 \\
MELD score & $0.642(0.569-0.724)$ & 0.000 & $0.718(0.546-0.943)$ & 0.017 \\
\hline
\end{tabular}

Note: $P<0.05$ was considered statistically significant.

Abbreviations: OR, odds ratio; $\mathrm{Cl}$, confidence interval; TBil, total bilirubin; INR, standardized ratio of prothrombin; ALB, plasma albumin; CR, serum creatinine; NA, serum sodium ions; MELD, model of end-stage liver disease.

$33 \%$ for chronic liver failure, ${ }^{22}$ and $62 \%$ for acute liver failure. ${ }^{25}$ The level of peripheral blood cortisol decreased with the aggravation of liver failure, and the incidence of RAI gradually increased $(P<0.05)$. The change of total serum cortisol is a reliable measure of RAI in patients with liver disease. RAI is associated with an increase in the cumulative incidence of mortality in all patients with severe liver disease such as ACLF. ${ }^{36}$ The condition of patients with end-stage liver disease could be accurately evaluated by the MELD score, which is positively correlated with mortality. ${ }^{26}$ The level of cortisol decreased as the MELD score increased. This suggested that cortisol levels may be one of the indicators to reflect the severity of liver failure. Cortisol levels in the no-survival group were significantly lower than that in the survival group at

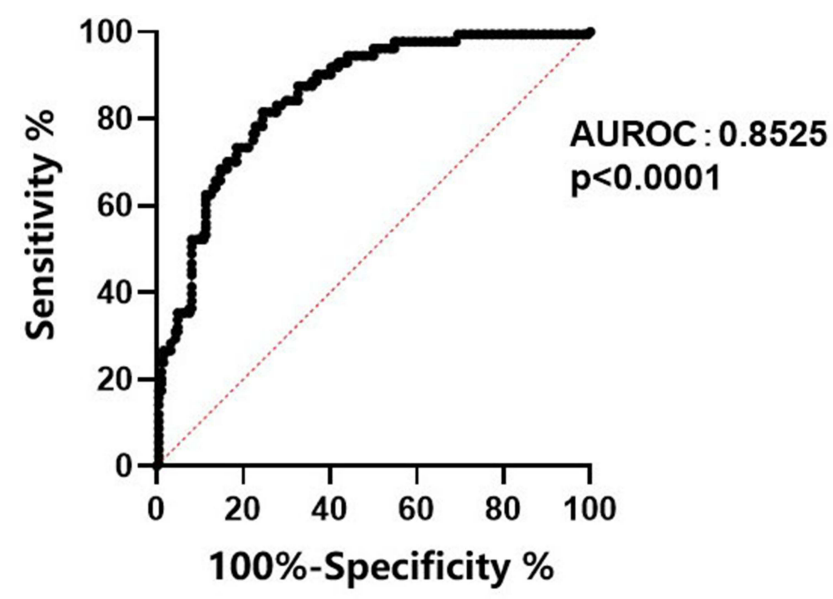

Figure 3 ROC: Receiver operating characteristics sensitivity and specificity for mortality based on cortisol level cut off $252.3 \mathrm{nmol} / \mathrm{L}$.
90 days after admission. Previous studies have shown that cortisol level is associated with the prognosis of liver failure, chronic liver disease, and decompensated cirrhosis and have suggested that as cortisol levels decline, the mortality of patients increases. ${ }^{24,27-29}$ The results of binary logistic regression analysis showed that age, MELD score and TBIL were independent protective factors affecting the prognosis of patients with liver failure at 90 days, and cortisol level was an independent risk factor. This study suggested that cortisol may be a useful predictor of the 90-day prognosis for liver failure.

Cortisol is one of the most important glucocorticoids secreted by the adrenal cortex. ${ }^{30,31}$ The hypothalamicpituitary-adrenal (HPA) axis could be activated and secretes glucocorticoids due to the disease. In patients with liver failure, the HPA axis can be activated by various cytokines, and glucocorticoid secretion is increased. But HPA is inhibited by excessive TNF- $\alpha$ in patients, resulting in reducing glucocorticoid secretion. ${ }^{32}$ It has been reported that cortisol is one of the most important indicators to evaluate stress response, and cortisol level is correlated with cell damage and functional failure. ${ }^{33}$ Sun et al investigated the relationship between the level of cortisol in peripheral blood and the condition and prognosis of patients with chronic acute liver failure associated with $\mathrm{HBV}^{34}$ They found that the level of cortisol gradually decreased with the severity of liver failure. In addition, cortisol levels increased over time in the survival group and decreased over time in the no-survival group. Fan et al investigated the relationship between the level of cortisol in peripheral blood of patients with HBVassociated liver failure and the severity of the disease and 
prognosis. ${ }^{35}$ The results of their study suggested that the cortisol level in the early stage of liver failure group (17.97 $\pm 3.16 \mu \mathrm{g} / \mathrm{dL}$ ) was significantly higher than that in the medium-stage liver failure group $(11.01 \pm 2.94 \mu \mathrm{g} / \mathrm{dL})$ and the end-stage of liver failure group $(6.66 \pm 2.60 \mu \mathrm{g} / \mathrm{dL})$. They also observed the dynamic pattern of serum cortisol level in patients with $\mathrm{HBV}$-associated liver failure and found that cortisol levels in the survival group increased. They proposed that ALB, MELD, TC, and cortisol were independent risk factors for the prognosis of patients with HBV-associated ACLF. Although the object of this study is not exactly the same as other studies, our results are basically consistent with that of previous studies.

In this study, we found that the level of cortisol was correlated with the severity of liver failure, which makes it possible to use cortisol when evaluating the prognosis of patients with liver failure at 90 days. However, this study still has some limitations. First, this study was conducted with a limited sample size. Second, the dynamic changes of cortisol levels were not monitored to evaluate the prognosis of patients.

\section{Conclusion}

Generally, this study prospectively analysed the correlation between cortisol levels and liver failure severity and 90-day prognosis. Our results suggested that cortisol played a crucial role in the process and severity of liver failure, and the higher the level was, the higher the survival rate of patients was.

In the future, we will continue to increase the sample data of different aetiologies and diseases for further research.

\section{Abbreviations}

MELD, model for end-stage liver disease; ROC, receiver operating curve; OR, odds ratio; $\mathrm{CI}$, confidence intervals; HBV, hepatitis B virus; ALT, glutamate aminotransferase; AST, aspartate aminotransferase; TBIL, total bilirubin; CR, serum creatinine; ALB, albumin; NA, serum sodium; ACTH, adrenocorticotropic hormone; PT-INR, the international standardization of prothrombin time; RAI, relative adrenal insufficiency; HPA, hypothalamic-pituitary-adrenal; AUROC, area under the receiver operating characteristics.

\section{Data Sharing Statement}

The datasets used or analyzed during the current study are available from the corresponding author on reasonable request.

\section{Ethics Approval and Consent to Participate}

This study was conducted in accordance with the Declaration of Helsinki and approved by the ethics committee of Beijing Youan Hospital (ethical batch number: 2019-989), and informed consent was obtained from all participants.

\section{Consent for Publication}

All authors final approval of the version to be published.

\section{Author Contributions}

All authors contributed to data analysis, drafting or revising the article, have agreed on the journal to which the article will be submitted, gave final approval of the version to be published, and agree to be accountable for all aspects of the work.

\section{Funding}

1) Hepatic Failure and Artificial Liver Treatment Research Beijing Key Laboratory Fund: 2-03-02-BJYAH2017007; 2) National Science and Technology Major Project "Prevention and Treatment of Major Infectious Diseases such as AIDS and Viral Hepatitis": 2017ZX10302201004-002.

\section{Disclosure}

All of the authors had no any personal, financial, commercial, or academic conflicts of interest separately.

\section{References}

1. Liver Failure and Artificial group, CSaID, Chinese Mwdical Association, et al. Guideline for diagnosis and treatment of liver failure. Chin J Hepatol. 2019;27(1):18-26.

2. Tomita K, Chiba N, Ochiai S, et al. Prognostic value of future liver remnant LU15 index of $99 \mathrm{mTc}$-galactosyl serum albumin scintigraphy for predicting posthepatectomy liver failure. PLoS One. 2021;16(2): e0247675. doi:10.1371/journal.pone.0247675

3. Kanjo A, Ocskay K, Gede N, et al. Efficacy and safety of liver support devices in acute and hyperacute liver failure: a systematic review and network meta-analysis. Sci Rep. 2021;11(1):4189. doi:10.1038/ s41598-021-83292-Z

4. Sagawa T, Kogiso T, Ito T, et al. Hereditary Apolipoprotein A-1 Amyloidosis with Glu34Lys mutation treated by liver transplantation: a case report. Transplant Proc. 2021;53(4):1327-1332. doi:10.1016/j. transproceed.2020.11.012

5. Wang F, Sun W, Xiao Q, et al. Peripheral T lymphocytes predict the severity and prognosis in patients with HBV-related acute-on-chronic liver failure. Medicine. 2021;100(5):e24075. doi:10.1097/ MD.0000000000024075

6. Miao J, Guo L, Wang L, et al. [Study on the application value of MELD-Na, CLIF-C OFs, COSSH-ACLFs and NLR scoring systems in patients with hepatitis B virus related acute-on-chronic liver failure]. Zhonghua Wei Zhong Bing Ji Jiu Yi Xue. 2020;32(12):1496-1501. Chinese. doi:10.3760/cma.j.cn121430-20200720-00536 
7. Tong JJ, Zhao W, Mu XY, et al. Predictive value of the Chinese group on the study of severe hepatitis B-acute-on-chronic liver failure score in the short-term prognosis of patients with hepatitis B virus-related acute-on-chronic liver failure. Chin Med J. 2019;132(13):1541-1549. doi:10.1097/CM9.0000000000000298

8. Chen T, Yang Z, Choudhury AK, et al. Complications constitute a major risk factor for mortality in hepatitis B virus-related acute-onchronic liver failure patients: a multi-national study from the Asia-Pacific region. Hepatol Int. 2019;13(6):695-705. doi:10.1007/ s12072-019-09992-x

9. Chen F, Shi Y, Ran S, et al. Predicting 90-day mortality at admission and 7 days post-admission among patients with hepatitis B virus-related acute-on-chronic liver failure. $J$ Pak Med Assoc. 2021;71(1(A)):22-27. doi:10.47391/JPMA.529

10. Huang $Z$, Wang $N$, Huang $S$, et al. Increased serum soluble urokinase plasminogen activator receptor predicts short-term outcome in patients with hepatitis B-related acute-on-chronic liver failure. Gastroenterol Res Pract. 2019;2019:3467690. doi:10.1155/2019/ 3467690

11. Dupuis C, Sabra A, Patrier J, et al. Burden of pneumococcal pneumonia requiring ICU admission in France: 1-year prognosis, resources use, and costs. Crit Care. 2021;25(1):24. doi:10.1186/ s13054-020-03442-z

12. Maepa SW, Ndlovu H. Advances in generating liver cells from pluripotent stem cells as a tool for modeling liver diseases. Stem Cells. 2020;38(5):606-612. doi:10.1002/stem.3154

13. May P, Normand C, Del Fabbro E, et al. Economic analysis of hospital palliative care: investigating heterogeneity by noncancer diagnoses. MDM Policy Pract. 2019;4(2):2381468319866451. doi:10.1177/2381468319866451

14. Fortea JI, Puente Á, Cuadrado A, et al. Congestive Hepatopathy. Int J Mol Sci. 2020;21(24):9420. doi:10.3390/ijms21249420

15. Qiang L, Qin J, Sun C, et al. A novel predictive model based on inflammatory markers to assess the prognosis of patients with HBV-related acute-on-chronic liver failure: a retrospective cohort study. BMC Gastroenterol. 2020;20(1):301. doi:10.1186/s12876020-01437-2

16. Hu C, Huang $\mathrm{K}$, Zhao $\mathrm{L}$, et al. Serum ammonia is a strong prognostic factor for patients with acute-on-chronic liver failure. Sci Rep. 2020;10(1):16970. doi:10.1038/s41598-020-73603-1

17. Guo HY, Zhang ZG, Zhang YY, et al. Risks and predicting factors of bleeding complications in hepatitis B virus-related acute-on-chronic liver failure. Turk J Gastroenterol. 2020;31(9):620-625. doi:10.5152/ tjg.2020.19307

18. Schulz M, Trebicka J. Acute-on-chronic liver failure: a global disease. Gut. 2021:gutjnl-2020-323973. doi:10.1136/gutjnl-2020323973

19. Coursin DB, Wood KE. Corticosteroid supplementation for adrenal insufficiency. JAMA. 2002;287(2):236-240. doi:10.1001/ jama.287.2.236

20. Subhani M, Dong V, Connolly A, et al. Trimethoprim-induced drug reaction with eosinophilia and systemic symptoms (DRESS) associated with reactivation of human herpes virus-6 (HHV-6) leading to acute liver failure. Clin Case Rep. 2020;8(12):2568-2573. doi:10.1002/ccr3.3218

21. Husebye Eystein S, Pearce SH, Krone,NP, et al. Adrenal insufficiency. Lancet. 2021;397(10274):613-629. doi:10.1016/ S0140-6736(21)00136-7
22. Marik PE. Adrenal insufficiency: the link between low apolipoprotein A-1 levels and poor outcome in the critically ill? Crit Care Med. 2004;32(9):1977-1978. doi:10.1097/01.ccm.0000132895.89019.32

23. Marik PE, Pastores SM, Annane D, et al. Recommendation for the diagnosis and treatment of corticosteroid insufficiency in critically ill adult patients: consensus statements from an international task force by the American College of Critical Care Medicine. Crit Care Med. 2008;36(6):1937-1949. doi:10.1097/CCM.0b013e31817603ba

24. Jie FR, Zheng RJ, Deng ZR, et al. Clinical significance of peripheral cortisol level in patients with hepatitis B virus-related acute-onchronic liver failure. Clin J Hepatol. 2017;25(5):383-385. doi:10.3760/cma.j.issn.1007-3418.2017.05.015

25. Harry R, Auzinger G, Wendon J. The clinical importance of adrenal insufficiency in acute hepatic dysfunction. Hepatology. 2012;36 (2):395-402. doi:10.1053/jhep.2002.34514

26. Kamath PS, Wiesner RH, Mailinchoc M, et al. A model to predict survival in patients with end-stage liver disease. Hepatology. 2001;33 (2):464-470. doi:10.1053/jhep.2001.22172

27. Pe M. Adrenal-exhaustion syndrome in patients with liver disease. Intensive Care Med. 2006;32(2):275-280. doi:10.1007/s00134-005$0005-5$

28. Jang JY, Kim TY, Sohn JH, et al. Relative adrenal insufficiency in chronic liver disease: its prevalence and effects on long-term mortality. Aliment Pharmacol Ther. 2014;40(7):819. doi:10.1111/apt.12891

29. Acevedo J, Fernández J, Prado V, et al. Relative adrenal insufficiency in decompensated cirrhosis: relationship to short-term risk of severe sepsis, hepatorenal syndrome, and death. Hepatology. 2013;58(5):1757-1765. Erratum in: Hepatology. 2015;61(6):2121. doi: 10.1002/hep.26535

30. Atwood RE, Golden DM, Kaba SA, et al. Characterization of the cortisol response to traumatic hemorrhage and intra-abdominal contamination models in cynomologus macaques. Mol Cell Endocrinol. 2020;518:111036. doi:10.1016/j.mce.2020.111036

31. Sonoda N, Takahata K, Tarumi W, et al. Changes in the cortisol and oxytocin levels of first-time pregnant women during interaction with an infant: a randomized controlled trial. BMC Pregnancy Childbirth. 2021;21(1):162. doi:10.1186/s12884-021-03609-8

32. Nazzari S, Fearon P, Rice F, et al. Beyond the HPA-axis: exploring maternal prenatal influences on birth outcomes and stress reactivity. Psychoneuroendocrinology. 2019;101:253-262. doi:10.1016/j. psyneuen.2018.11.018

33. Marcato F, van den Brand H, Jansen CA, et al. Effects of pre-transport diet, transport duration and transport condition on immune cell subsets, haptoglobin, cortisol and bilirubin in young veal calves. PLoS One. 2021;16(2):e0246959. doi:10.1371/journal.pone.0246959

34. Sun YQ, Chang SZ, Shen F, et al. Relationship between cortisol levels in peripheral blood and prognosis in elderly patients with chronic acute liver failure associated with hepatitis B virus. Chin $J$ Geriatr. 2015;35(4):944-945.

35. Fan CC, Zhao CY, Wang YD, et al. Changes of cortisol levels in peripheral blood in patients with chronic hepatitis B virus associated acute liver failure. Chin J Infect Dis. 2013;06:353-357.

36. Piano S, Favaretto E, Tonon $M$, et al. Including relative adrenal insufficiency in definition and classification of acute-on-chronic liver failure. Clin Gastroenterol Hepatol. 2020;18(5):1188-1196.e3. doi:10.1016/j.cgh.2019.09.035 


\section{Publish your work in this journal}

Risk Management and Healthcare Policy is an international, peerreviewed, open access journal focusing on all aspects of public health, policy, and preventative measures to promote good health and improve morbidity and mortality in the population. The journal welcomes submitted papers covering original research, basic science, clinical \& epidemiological studies, reviews and evaluations, guidelines, expert opinion and commentary, case reports and extended reports. The manuscript management system is completely online and includes a very quick and fair peer-review system, which is all easy to use. Visit http://www.dovepress.com/testimonials.php to read real quotes from published authors.

Submit your manuscript here: https://www.dovepress.com/risk-management-and-healthcare-policy-journal 